\title{
IDENTIFICANDO LOS CONOCIMIENTOS PARA ENSEÑAR MATEMÁTICAS EN EDUCACIÓN INFANTIL: UN PRIMER PASO PARA EL DESARROLLO PROFESIONAL
}

\section{IDENTIFYING KNOWLEDGE FOR TEACHING MATHEMATICS IN EARLY CHILDHOOD EDUCATION: A FIRST STEP FOR PROFESSIONAL DEVELOPMENT}

\author{
Ángel Alsina \\ Universidad de Girona - España \\ angel.alsina@udg.edu \\ Rosa Delgado \\ Pontificia Universidad Católica de Valparaíso - Chile \\ rosamdelgadorebolledo@gmail.com
}

\section{Resumen}

En este estudio se indaga en las prácticas de enseñanza de las matemáticas del profesorado de Educación Infantil, asumiendo que la identificación de los componentes de dicha práctica es el punto de partida necesario para promover el desarrollo profesional. En este sentido, se han identificado los conocimientos para enseñar matemáticas que pone en juego una maestra durante una sesión de clases, a partir de los dominios y subdominios que componen el modelo de Conocimientos para Enseñar Matemáticas en Educación Infantil (CEM-EI). El análisis ha permitido identificar evidencias de los tres subdominios del Conocimiento Matemático en Educación Infantil (CM-EI): Conocimientos matemáticos Intuitivos e Informales (C-IeI); Conocimiento de los contenidos matemáticos (C-CM) y Conocimiento de los procesos matemáticos (C-PM), y dos de los tres subdominios del Conocimiento Didáctico de las Matemáticas en Educación Infantil (CDM-EI): Conocimiento sobre las formas de aprendizaje de las matemáticas en la infancia (C-FAM) y Conocimiento sobre la planificación y gestión de actividades de enseñanza de las matemáticas (C-PGA). Se concluye que serán necesarios nuevos estudios que permitan afinar y consolidar estos primeros resultados.

Palabras-clave: conocimiento del profesorado para enseñar matemáticas, prácticas de enseñanza de las matemáticas, profesorado de Educación Infantil

\begin{abstract}
This study investigates the mathematics teaching practices of Early Childhood Education teachers, assuming that the identification of the components of this practice is the necessary starting point to promote the professional development of teachers. From this point of view, we have identified the mathematics teaching knowledge of an Early Childhood Education teacher during a class session,
\end{abstract}


based on the domains and subdomains of the model of Knowledge for Teaching Mathematics in Early Childhood Education (CEM-EI, by its Spanish acronym), which describes the specific knowledge needed by teachers at this stage to teach mathematics. The analysis identified evidence of the three subdomains of Mathematical Knowledge in Early Childhood Education (CM-EI): Intuitive and Informal Mathematical Knowledge (C-IeI); Knowledge of Mathematical Content (C$\mathrm{CM}$ ) and Knowledge of Mathematical Processes (C-PM), and two of the three subdomains of Mathematical Pedagogical Knowledge in Early Childhood Education (CDM-EI): Knowledge about ways of learning mathematics in early childhood (C-FAM) and Knowledge about planning and managing mathematics teaching activities (C-PGA). It is concluded that further studies will be necessary to refine and consolidate these initial results.

Keywords: teachers' knowledge for teaching mathematics, mathematics teaching practices, Early Childhood Education teachers

\section{INTRODUCCIÓN}

Desde hace varias décadas se ha ido incrementando el interés por el conocimiento del profesorado para enseñar matemáticas debido, entre otras razones, a las repercusiones que este conocimiento tiene en el aprendizaje del alumnado. Esta es una cuestión muy relevante, ya que, si el profesorado no tiene un conocimiento matemático y didáctico apropiado, el proceso de enseñanza de las matemáticas puede verse debilitado y, por consiguiente, el aprendizaje del alumnado seriamente amenazado.

Por esta razón, desde que Shulman (1986) objetivó los diversos tipos de conocimiento del profesorado para enseñar las asignaturas del currículum escolar, dentro de la agenda de investigación sobre el desarrollo profesional del profesorado de matemáticas han surgido diversos modelos que describen los conocimientos del profesorado para enseñar matemáticas. Destacan, por ejemplo, el Knowledge Quartet (KQ), que considera cuatro categorías que permiten observar, analizar y discutir acerca de situaciones de aula en las que el conocimiento del profesorado se pone en acción (ROWLAND; HUCKSTEP; THWAITES, 2005); el Mathematical Knowledge for Teaching (MKT), que considera un conjunto de conocimientos y habilidades que requiere el profesorado para gestionar las tareas y los problemas recurrentes en la enseñanza de las matemáticas (BALL; THAMES; PHELPS, 2008); el Modelo de Conocimientos y Competencias Didáctico-Matemáticas (CCDM), para analizar, interpretar, caracterizar y categorizar los conocimientos que pone en juego el profesorado al enseñar un determinado contenido matemático (GODINO et al., 2017); o el Modelo del Conocimiento Especializado del Profesor de Matemáticas (MTSK), que se interesa por el conocimiento que es útil para el profesor en el contexto de enseñanza y aprendizaje de las matemáticas 
(CARRILLO et al., 2018).

Este estudio se focaliza en los conocimientos para enseñar matemáticas en Educación Infantil, asumiendo que se trata de una etapa imprescindible del desarrollo humano, en la que se establecen las bases para los aprendizajes posteriores. Desde este punto de vista, el profesorado de infantil debería disponer de conocimientos tanto matemáticos como didácticos que le permitan promover el aprendizaje en esta etapa y favorecer las relaciones de los niños con la asignatura de matemáticas en niveles educativos posteriores. No obstante, coexisten, como mínimo, dos obstáculos alrededor de los conocimientos para enseñar matemáticas en Educación Infantil: el primero, tiene que ver con la adquisición de estos conocimientos profesionales y, el segundo, con los modelos de análisis para identificarlos.

En relación al primero de estos inconvenientes, diversos estudios recientes revelan que la formación inicial que recibe el futuro profesorado de Educación Infantil sobre matemáticas y su didáctica es muy escasa (ALSINA, 2020a; NOLLA et al., 2021). Alsina (2020a), por ejemplo, analiza la presencia de las asignaturas de Matemáticas y de Didáctica de las Matemáticas en el Grado de Maestro de Educación Infantil en España y compara estos datos con los de otros países como Argentina, Colombia, Chile, Ecuador y México. El autor expone que la tendencia mayoritaria en las universidades españolas analizadas es que se dedica alrededor de un 2,5\% del total de créditos/horas a Didáctica de las Matemáticas, mientras que la Matemática prácticamente no está presente. En las mallas curriculares de las universidades latinoamericanas se observa una mayor variabilidad en relación con los créditos/horas destinadas a la Didáctica de las Matemáticas (desde el 1,8\% hasta el 6,25\%), pero en todas ellas la cantidad de créditos destinados a las Matemáticas es superior. Nolla et al. (2021), en un estudio realizado con datos de todas las universidades españolas, constatan además la escasa existencia de menciones en matemáticas y su didáctica en la formación inicial del profesorado. La limitada formación en este ámbito que recibe el futuro profesorado es pues una amenaza muy importante, ya que como indican Heargreaves et al. (2001) "si el profesor no sabe cómo hacerlo o a la hora de la verdad no se siente seguro haciéndolo, no se puede hacer", es decir, no se puede enseñar bien lo que no se sabe.

Más allá de poner de manifiesto la situación actual acerca de la escasa presencia 
tanto de la Didáctica de las Matemáticas como de las Matemáticas en el Grado de Maestro de Educación Infantil, junto con reivindicar que es necesaria una Agenda para la Acción que permita mejorarla (ALSINA, 2020a), el propósito de este estudio es indagar en los conocimientos para enseñar matemáticas en Educación Infantil. En este ámbito, existe otro impedimento que tiene que ver con el hecho de que la mayoría de estudios que se han realizado desde los distintos modelos de conocimiento del profesorado de matemáticas descritos (BALL; THAMES; PHELPS, 2008; CARRILLO et al., 2018; GODINO et al., 2017; ROWLAND; HUCKSTEP; THWAITES, 2005) se han llevado a cabo con profesorado de las etapas de primaria y secundaria, mientras que las investigaciones sobre el conocimiento del profesorado de infantil son más bien escasas (CHARALAMBOUS y PITTA-PANTAZI, 2016), aunque se reconoce la importancia de poder caracterizar tanto el conocimiento como las debilidades en el conocimiento de los profesores de infantil en diferentes temas matemáticos, vinculando el conocimiento con otros elementos con la competencia mirar profesionalmente (e.g., Samuel, Vanegas y Jiménez, 2018). Adicionalmente, algunos de los dominios y subdominios que se incluyen en los modelos de conocimiento no se ajustan a las características específicas de la etapa de Educación Infantil, razón por la cual Alsina y Delgado (en revisión) desarrollan el modelo de "Conocimientos para Enseñar Matemáticas en Educación Infantil" (CEM-EI) que está constituido por dos dominios: Conocimiento Matemático en Educación Infantil (CM-EI) y Conocimiento Didáctico de las Matemáticas en Educación Infantil (CDM-EI).

El CEM-EI es un modelo de análisis que describe los conocimientos del profesorado de Educación Infantil para enseñar matemáticas. Desde este marco, el objetivo de este artículo es identificar los conocimientos para enseñar matemáticas que pone en juego una maestra de Educación Infantil durante una sesión de clases, a partir de los dominios y subdominios que componen el CEM-EI. Con ello, se pretende también iniciar un proceso de refinamiento de los elementos que componen el modelo.

\section{MODELO DE CONOCIMIENTOS PARA ENSEÑAR MATEMÁTICAS EN EDUCACIÓN INFANTIL (CEM-EI)}

A partir de una revisión exhaustiva sobre la aplicación de los modelos de conocimiento del profesorado de matemáticas a la Educación Infantil, Alsina y Delgado 
(en revisión) subrayan que algunos componentes de estos modelos no se ajustan a dicha etapa. Por ejemplo, debido a que en infantil no es tan usual contar con un libro de texto, sería poco útil considerar como parte del conocimiento del profesorado de esta etapa elementos asociados al uso de libros de texto para la enseñanza. Para responder a esta dificultad de aplicabilidad, subrayan tres estrategias posibles: 1) modificar los modelos de conocimiento del profesorado de matemáticas para que sean más cercanos al conocimiento del profesorado de infantil; 2) utilizar los modelos existentes profundizando en aquellos componentes que identifican al profesorado de infantil; 3) desarrollar modelos de conocimiento específicos del profesorado de infantil.

Considerando esta última estrategia, Alsina y Delgado (en revisión) diseñan el modelo CEM-EI, junto con describir exhaustivamente el proceso de construcción. Muy sintéticamente, para diseñar dicho modelo se han examinado a fondo los escasos estudios existentes sobre el conocimiento de los profesores de Educación Infantil (e.g., MOSVOLD ET AL., 2011; HUNDELAND ET AL., 2017; MUÑOZ-CATALÁN ET AL., 2019). A partir de esta revisión, se ha identificado que los estudios analizados coinciden en describir conocimientos respecto a los contenidos matemáticos, el uso del lenguaje matemático, conexiones entre contenidos, cómo enseñar matemáticas y cómo aprende el alumnado. Lo anterior se justifica oportunamente en el estudio indicado. Adicionalmente, se han considerado las características específicas de la enseñanza de las matemáticas en Educación Infantil a partir de la revisión de Alsina (2020b), donde se describen las finalidades de la enseñanza (¿para qué se enseña? y ¿por qué se enseña?), las prácticas de enseñanza (¿cómo se enseña?) y la organización de la enseñanza (¿cuándo se enseña? y ¿qué se enseña?). En la Figura 1 se muestran los dominios del modelo CEM-EI y sus respectivos subdominios. 
Figura 1 - Modelo de Conocimientos para Enseñar Matemáticas en Educación Infantil

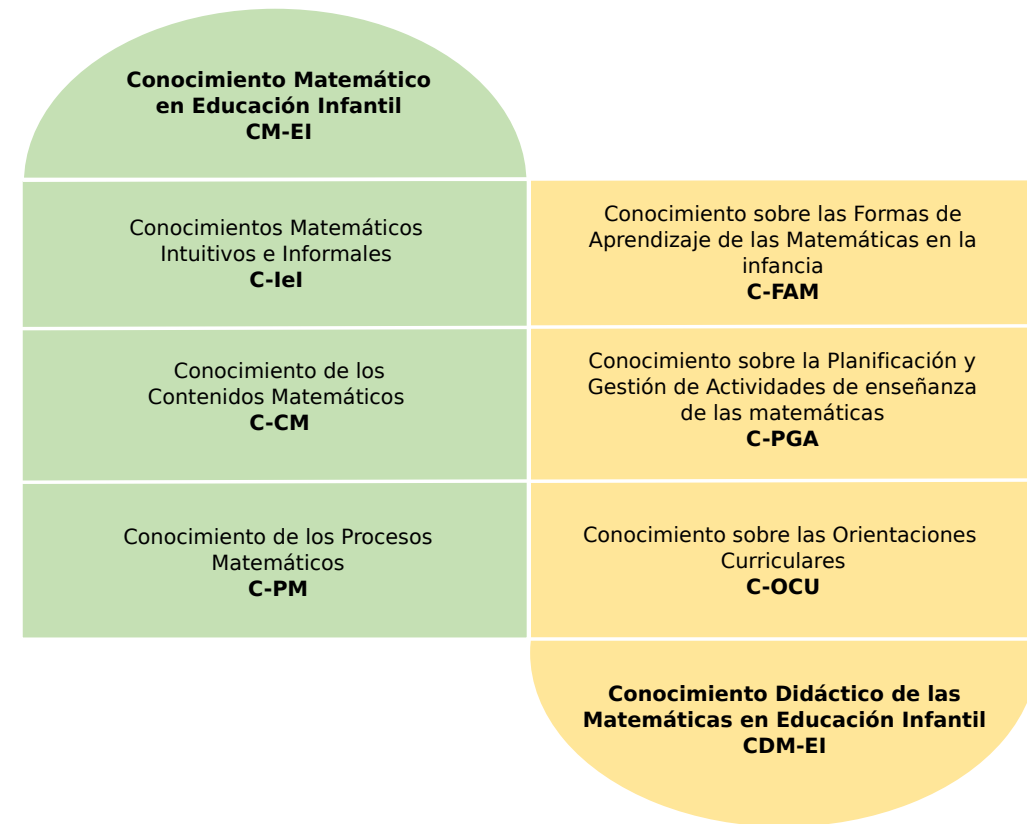

Fuente: Alsina y Delgado (en revisión)

\section{El Conocimiento Matemático en Educación Infantil (CM-EI)}

La formulación de este dominio de conocimiento asume que el profesorado de infantil requiere un conocimiento matemático específico y estructurado que les permita promover el desarrollo de conocimientos y habilidades matemáticas de los niños menores de 6 años. El CM-EI está formado por tres subdominios (Figura 1):

Conocimientos matemáticos Intuitivos e Informales (C-IeI): Se refiere a las primeras matemáticas que los niños aprenden y usan en el marco de experiencias informales como la exploración del entorno, la manipulación y experimentación con materiales diversos y el juego (BAROODY, 1987). Las matemáticas intuitivas e informales han sido objeto de análisis de diversos autores, entre ellos Alsina (2015), quien organiza las acciones de los niños asociadas a estas matemáticas en cuatro categorías: las cualidades sensoriales, las cantidades continuas y discretas, las posiciones y formas y, finalmente, los atributos mesurables. Así, las primeras nociones espaciales, los primeros aspectos cuantitativos que los niños identifican, las distintas sensaciones que experimentan al tocar objetos de diversos materiales y texturas, algunas características referentes a atributos mesurables como la masa o la capacidad, la estimación de cantidades y la enumeración son ejemplos de matemáticas intuitivas e informales (ALSINA y MARTÍNEZ, 2016). En línea 
con lo anterior, el subdominio C-IeI se refiere al conocimiento del profesorado de infantil sobre las matemáticas intuitivas que los niños aprenden en situaciones informales, además del papel que éstas juegan en el desarrollo de su pensamiento matemático.

Conocimiento de los contenidos matemáticos (C-CM): Se refiere al conocimiento del profesorado de infantil sobre las matemáticas que los niños aprenden en el contexto escolar. Diversos organismos y autores han descrito y analizado los contenidos matemáticos que contribuyen a desarrollar el pensamiento matemático de los niños durante la etapa de Educación Infantil (ALSINA, 2006, 2019a; CLEMENTS y SARAMA, 2009; GEIST, 2014; NCTM, 2000; entre otros). El NCTM (2000), por ejemplo, explicita los estándares de contenido que se deberían incluir en los programas de enseñanza desde los 3 años para capacitar a todos los estudiantes: números y operaciones, álgebra, geometría, medida, y análisis de datos y probabilidad. Además, sugiere la atención que deberían recibir los distintos estándares en las diversas etapas.

Alsina (2019a) organiza y desglosa los contenidos correspondientes a esto cinco estándares para la Educación Infantil. Por ejemplo, los contenidos de magnitud y medida incluyen la identificación de magnitudes continuas, las agrupaciones, clasificaciones y ordenaciones de elementos según su medida, la composición y descomposición de medidas, y las estimaciones y cuantificación. Considerando lo anterior, el conocimiento de los contenidos matemáticos y el conocimiento de relaciones entre contenidos matemáticos, forman parte del subdominio C-CM.

Conocimiento de los procesos matemáticos (C-PM): Los procesos matemáticos ponen de relieve las formas de adquisición y uso de los contenidos matemáticos (NCTM, 2000). Desde este planteamiento, se considera que para construir conocimiento y desarrollar habilidades matemáticas se requiere la realización de procesos como resolver problemas, modelizar, razonar, argumentar, comunicar, representar, etc. Los estudios acerca de la enseñanza de las matemáticas a través de estos procesos hacen hincapié en el desarrollo de habilidades como pensar y hacer, más que memorizar definiciones y procedimientos (ALSINA et al., 2021). En esta línea, aunque muchos de estos estudios se han focalizado en las etapas de primaria y secundaria, algunos de ellos han incidido también en la etapa de infantil (ALSINA, 2012; CROSS; WOODS; SCHWEINGRUBER, 2009; CLEMENTS \& SARAMA, 2009; entre otros). 
ALSINA (2012), por ejemplo, subraya que la resolución de problemas se plantea en forma de juegos que se introducen con una serie de reglas y se van adquiriendo técnicas y estrategias que conducen al éxito. Por su parte el razonamiento y la demostración están presentes en actividades de relación o comparación a través de clasificaciones, ordenaciones o correspondencias. La comunicación aparece en el uso progresivo del léxico adecuado, la expresión de ideas de manera oral y la escucha a los demás. Por su parte, la representación se observa en las formas de expresión gráfica y el inicio de la notación escrita de los niños. En esta línea, el conocimiento del profesorado de infantil de qué es un problema matemático y qué cuenta como un problema en este nivel, las estrategias y heurísticas de resolución de problemas que se pueden utilizar; cómo, porqué y para qué se argumenta en el aula de infantil; cuáles son los diferentes tipos de razonamientos que se ponen en juego; cómo se utiliza el lenguaje para comunicar ideas matemáticas o bien qué representaciones de las ideas matemáticas se pueden realizar, son elementos que forman parte del subdominio de C-PM.

\section{El Conocimiento Didáctico de las Matemáticas en Educación Infantil (CDM-EI)}

Este dominio de conocimiento incluye tres subdominios acerca de los conocimientos psicopedagógicos y didácticos sobre cómo aprenden matemáticas los niños y cómo se enseñan las matemáticas en Educación Infantil (Figura 1):

Conocimiento sobre las formas de aprendizaje de las matemáticas en la infancia (C-FAM): La investigación educativa ha descrito un amplio conjunto de conocimientos sobre las formas de aprender durante la infancia (e.g., Malaguzzi, 2001; Montessori, 1986; Piaget, 1975; Pikler, 1985; entre otros). Estos estudios han determinado que los niños empiezan a desarrollar su pensamiento matemático a partir de la visualización de las ideas matemáticas de manera concreta, las situaciones reales y el uso de manipulativos, principalmente. Progresivamente, son capaces de representar mentalmente el conocimiento y avanzar hacia la esquematización y formalización.

Más recientemente, se han aportado también conocimientos desde la neurociencia (BROWN, 2018), que añaden fundamento científico a lo que estos autores clásicos han investigado e implementado durante años a través de la observación, la documentación pedagógica y el reajuste educativo a la infancia. Estos estudios, en su conjunto, se refieren a la importancia de los primeros años para el desarrollo y el aprendizaje posterior, la 
plasticidad del cerebro, la estrecha relación entre el desarrollo cognitivo, afectivo y social, o bien el desarrollo de la autorregulación cognitiva y emocional, entre otros aspectos. Este conjunto de conocimientos sobre cómo aprenden matemáticas los niños menores de 6 años y qué elementos influyen en este aprendizaje se incluyen en el subdominio C-FAM.

Conocimiento sobre la planificación y gestión de actividades de enseñanza de las matemáticas (C-PGA): En el Enfoque de los Itinerarios de Enseñanza de las Matemáticas (EIEM), Alsina $(2018,2019 b, 2020 c)$ plantea la planificación de las actividades de enseñanza a partir de itinerarios en forma de secuencias intencionadas que contemplan tres niveles: 1) contextos informales, que permiten visualizar las ideas matemáticas de manera concreta (situaciones de vida cotidiana, materiales manipulativos y juegos); 2) contextos intermedios, que conducen a la esquematización y generalización progresiva del conocimiento matemático (recursos literarios y tecnológicos); y 3) contextos formales, en los que se trabaja la representación y formalización del conocimiento matemático con procedimientos y notaciones convencionales (recursos gráficos), para completar de esta forma el aprendizaje desde lo concreto hasta lo simbólico.

En relación a la gestión, este mismo autor propone implementar prácticas productivas, asumiendo que una práctica productiva en Educación Matemática es una acción o destreza educativa útil y provechosa para promover el aprendizaje de las matemáticas con sentido en todos los niveles (ALSINA, 2020d). Desde esta perspectiva, describe cinco prácticas productivas que se fundamentan en la enseñanza de los contenidos matemáticos a través de los procesos: 1) promover la resolución de situaciones problemáticas que impliquen pensar; 2) plantear preguntas efectivas en la clase de matemáticas que impliquen argumentar; 3) fomentar la comunicación en el aula de matemáticas en un ambiente que invite a interactuar, negociar y dialogar; 4) diseñar e implementar actividades matemáticas que requieran hacer conexiones; y 5) incentivar la expresión oral, gráfica y/o simbólica de las ideas matemáticas internas y las acciones externas a través de tareas que impliquen representar. Este conjunto de conocimientos sobre el diseño y la gestión de actividades que permitan promover una educación matemática inclusiva en Educación Infantil se exponen en el C-PGA.

Conocimiento sobre las orientaciones curriculares (C-OCU): En las últimas décadas, y como consecuencia del auge de la educación matemática infantil, diversos 
organismos de prestigio internacional han hecho recomendaciones sobre cómo se deberían plantear las matemáticas en los currículums de los primeros niveles escolares. Por ejemplo, en Early childhood mathematics: Promoting good beginnings (NAEYC \& NCTM, 2002), se propone por ejemplo, basar los currículos de matemáticas y las prácticas docentes en el conocimiento sobre el desarrollo cognitivo, lingüístico, físico, social y emocional de los niños; utilizar currículos y prácticas docentes que fortalezcan los procesos infantiles de resolución de problemas y razonamiento, así como los de representación, comunicación y conexión de ideas matemáticas; asegurar que el currículo sea coherente y compatible con las relaciones y secuencias conocidas de las ideas matemáticas fundamentales; o apoyar el aprendizaje mediante la evaluación continua y reflexiva del conocimiento, destrezas y estrategias de todos los niños.

En síntesis, el conocimiento acerca de la organización curricular de la Educación Infantil (que en muchos países se estructura áreas, en lugar de asignaturas), la presencia de los conocimientos matemáticos en la organización curricular, o la evaluación (inicial y formativa, principalmente) como elemento indisociable del proceso de enseñanzaaprendizaje, forman parte del C-OCU.

\section{CONTEXTO Y MÉTODO}

Desde un enfoque cualitativo y un paradigma interpretativo, en esta investigación nos proponemos identificar el conocimiento para enseñar matemáticas en Educación Infantil. Nos aproximamos a dicho conocimiento través de un estudio de caso (Ponte, 2006). El caso seleccionado es una maestra cuyas clases están dirigidas a niños y niñas de 5-6 años en el Colegio de Sigüeiro, en Oroso (A Coruña, España). La maestra, que de ahora en adelante llamaremos Sara, es además Doctora en Didáctica de las Matemáticas y posee 16 años de experiencia en Educación Infantil. Sara ha mostrado interés por analizar su práctica de enseñanza y mejorarla continuamente, a la vez que se apoya de algunos marcos teóricos en Educación Matemática para la planeación y realización de sus clases.

Los datos del estudio provienen de una sesión de clases en la que, a partir de comentarios sobre la estación del año y el tiempo meteorológico del día anterior, Sara genera una discusión con los estudiantes sobre la temperatura del agua de lluvia. Posteriormente, la maestra propone un problema cuya resolución requiere movilizar 
conocimientos matemáticos asociados a la medición, la clasificación y la ordenación. La sesión de clase fue grabada en vídeo y luego transcrita incluyendo algunas imágenes de las producciones de los estudiantes, así como de lo escrito por Sara en la pizarra.

Para la interpretación de los datos desarrollamos un análisis de contenido (Krippendorff, 2004), de modo que la sesión de clases fue dividida en cuatro momentos: asamblea en gran grupo, exploración, presentación de los resultados, comprobación y argumentación. Esta división está acordé con la estructura que Sara manifiesta haber planeado para la clase. En cada uno de estos momentos se escogieron como unidades de análisis aquellos fragmentos de clase donde las intervenciones de Sara y las interacciones entre la maestra y sus estudiantes daban cuenta de un conocimiento que podía clasificarse en uno de los subdominios del modelo CEM-EI. De este modo, los descriptores específicos de cada subdominio se construyeron a partir de los datos obtenidos al observar la clase.

En la transcripción de los fragmentos de la clase los estudiantes que intervienen son nombrados con la inicial E acompañada de un número. Como ejemplo de lo anterior, presentamos un fragmento de clase donde las expresiones de la profesora que se destacan con negrita son aquellas que interpretamos como su conocimiento de la comparación como un contenido matemático en Educación Infantil.

Sara: ¿Ya mediste alguno?

$\mathrm{E} 2: \mathrm{Si}$

Sara: muy bien, me dices entonces lo que registrasteis

E2: Si profe, el de la nevera 1 y 0 , el de la lluvia un 2

Sara: ¿Sí?, ¿estáis seguros?

E3: Si 1 y 0 es 10 el frío, y el 2 templado

Sara: 10 ¿es más o menos que 2?

E3: Másssssssssss

\section{Sara: ¿Y entonces?, ¿cómo es [templado] un número más pequeño?}

De manera similar se analizaron otros fragmentos de clase y la pertenencia de las unidades de análisis a los subdominios del modelo fue discutida por ambos autores de este trabajo logrando coincidencias en la interpretación de los fragmentos de clase que se expondrán en la próxima sección. 


\section{RESULTADOS}

En esta sección, mostramos los subdominios del modelo CEM-EI identificados en el conocimiento de Sara durante el desarrollo de una clase sobre temperatura. Vale la pena mencionar que otras sesiones de clase de Sara fueron grabadas y analizadas, sin embargo, en esta investigación se presentan los resultados de aquella sesión donde la maestra puso en juego una variedad de conocimientos que permiten ejemplificar los diferentes subdominios del modelo CEM-EI.

Durante el momento de la asamblea en gran grupo, Sara se dirige a los estudiantes haciendo preguntas sobre el otoño y la lluvia del día anterior. Desde este contexto informal, Sara va dirigiendo la discusión hacía la temperatura que podría tener el agua de lluvia, concluyendo que es necesario hacer una medición. En el siguiente fragmento la discusión continua en la siguiente forma:

Sara: Ya, con el termómetro medimos y sabemos la temperatura del agua. Pero... ¿cómo sabemos si es fría?

E1: Cogemos con la de la nevera y miramos.

Sara: ¡Qué buena idea!, ¿Y caliente?

E1: Pues con la del microondas.

E1 sugiere considerar la temperatura que alcanza el agua en la nevera y la que alcanza el agua en el microondas como puntos de referencia para hacer comparaciones con la temperatura del agua de lluvia. Sara retoma esta idea dibujando en la pizarra cinco columnas como se representa en la Figura 3. La maestra tiene un balde con agua de lluvia e invita a los estudiantes a que toquen el agua y luego se acerquen a la pizarra ubicándose en el lugar que consideran está la temperatura del agua: más frío que en la nevera, como el agua de la nevera, menos fría que en la nevera y menos caliente que en el microondas, como en el microondas y más caliente que en el microondas.

Figura 3 - Puntos de referencia para estimar la temperatura

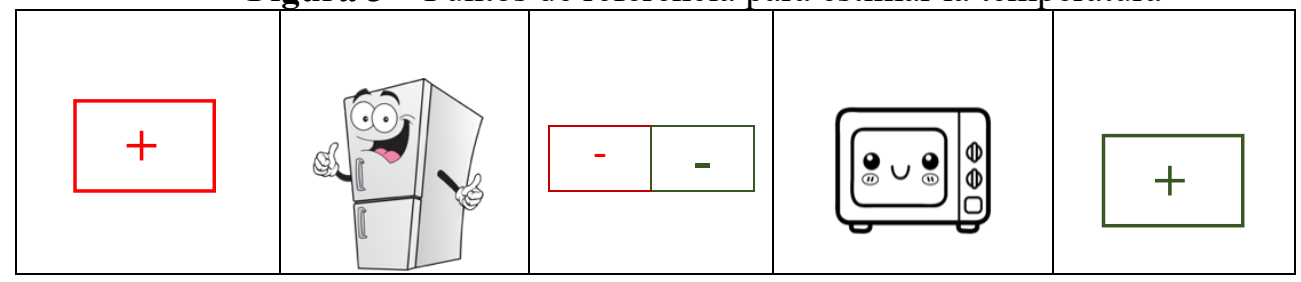

Fuente: elaboración propia 
La mayoría de los estudiantes se ubicaron en la columna correspondiente a una temperatura más fría que en la nevera, mientras que ninguno de ellos consideró que el agua estuviera más caliente que en el microondas. Esta ubicación en columnas hizo que los estudiantes formaran un diagrama de barras humano, que luego es representado por ellos dibujando sobre las columnas el número de cruces correspondientes a las elecciones del grupo. En este sentido, la profesora promueve el uso del diagrama de barras como representación de la situación discutida dando cuenta de su conocimiento de la representación como proceso matemático (C-PM). Sin embargo, dado que el diagrama de barras no es el elemento central de la clase, sino que hace parte de una actividad introductoria planteada desde un contexto informal, en este fragmento también se pone en juego el conocimiento de la profesora sobre la planificación y gestión de actividades de enseñanza de las matemáticas (C-PGA) respecto a incentivar la expresión gráfica de las ideas matemáticas de los estudiantes.

Figura 4 - Representación de las estimaciones de los estudiantes

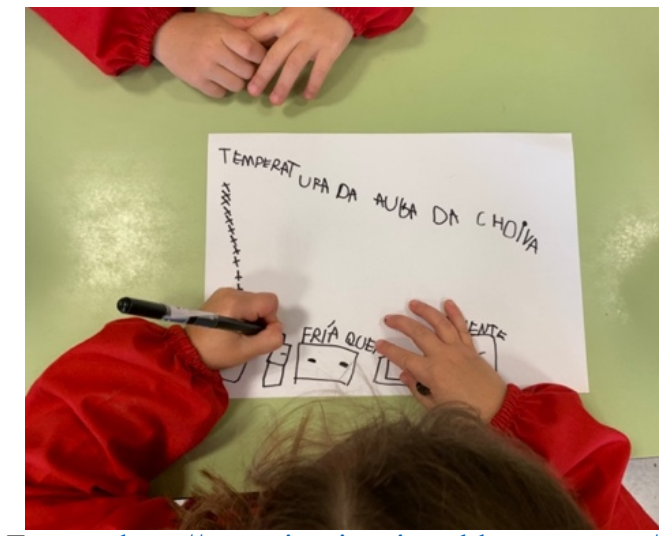

Fuente: http://eucocinoticocinas.blogspot.com/

En línea con lo anterior, también observamos que Sara guía a los estudiantes a que realicen una estimación de la temperatura del agua a partir de sus creencias y sensaciones. De este modo, identificamos su conocimiento de las cualidades sensoriales como parte de las matemáticas intuitivas e informales que sus estudiantes utilizan (C-IeI). Este conocimiento a su vez está relacionado con la comparación como un conocimiento matemático escolar y la representación como un proceso matemático.

Ahora, en el momento de exploración los niños se reúnen en grupos (de 5 o 6 miembros) y Sara entrega a cada grupo un termómetro, hojas de papel y un marcador, además de un vaso con agua de lluvia, otro con agua calentada en el microondas y otro con 
agua enfriada en la nevera. Sara solicita a los estudiantes que dibujen un termómetro en una de sus hojas, este dibujo debe irse completando con las temperaturas que se obtienen en cada medición (Ilustración 2).

Figura 5 - Materiales usados para realizar la tarea
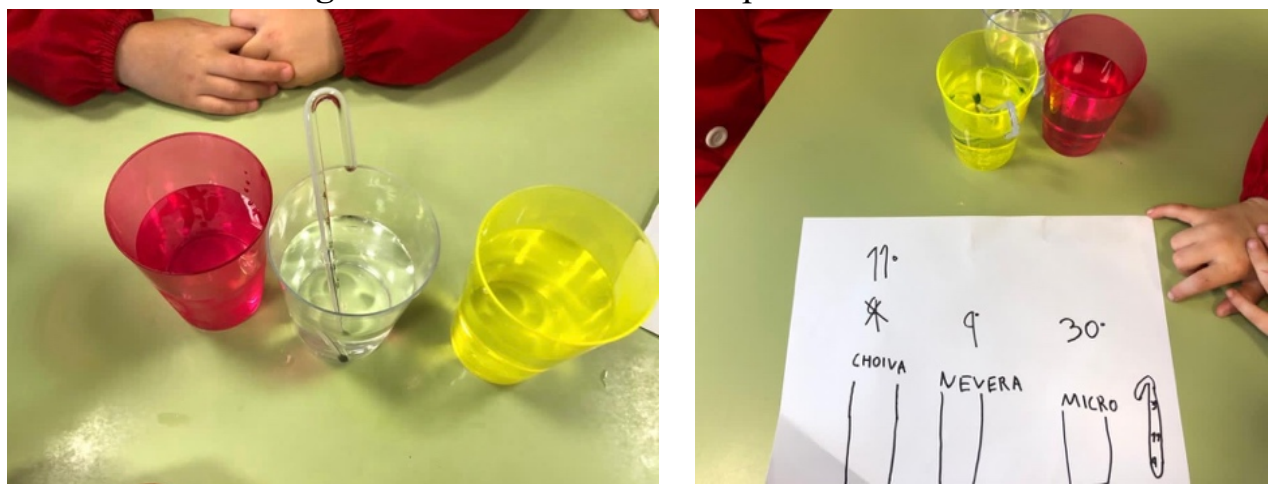

Fuente: http://eucocinoticocinas.blogspot.com/

Transcurrido un tiempo, la maestra se acerca a los grupos para resolver sus dudas sobre el trabajo que están realizando. En uno de los grupos se presenta la siguiente situación:

Sara: ¿Ya mediste alguno?

$\mathrm{E} 2: \mathrm{Si}$

Sara: muy bien, me dices entonces lo que registrasteis

E2: Si profe, el de la nevera 1 y 0 , el de la lluvia un 2

Sara: ¿Sí?, ¿estáis seguros?

E3: Si 1 y 0 es 10 el frío, y el 2 templado

Sara: 10 ¿es más o menos que 2?

E3: Másssssssssss

\section{Sara: ¿Y entonces?, ¿cómo es [templado] un número más pequeño?}

El episodio anterior continúa cuando el grupo que decide repetir la medición, obtiene como resultado 20 grados y concluye que el registro anterior era errado pues el 20 es más grande que el 10. Cuando Sara notó que los estudiantes no registraron correctamente la temperatura del agua de lluvia los llevó hacía la comparación numérica para darle otro sentido a la situación, de este modo la maestra promueve la construcción de conocimiento matemático respecto a la relación "mayor que" y "menor que". Así, identificamos en este fragmento el conocimiento de Sara de la comparación como un contenido matemático en Educación Infantil (C-CM). 
Continuando con la clase, Sara se acerca a otro grupo y observa que los resultados obtenidos fueron 10, 20 y 40 grados correspondientes al agua de la nevera, de la lluvia y del microondas respectivamente. Una de las estudiantes presenta los resultados del grupo de la siguiente forma:

E5: La de la nevera, es el número más pequeño.

Sara: ¿sí?

E5: Si de estos (señalando 10, 20 y 40)

Sara: Y los otros ¿qué os salió?

E5: Más grandes porque no está fría.

Sara: ¿más grandes? ¿mucho?

E5: El [número] del micro mucho, se sale del termómetro

Sara: ¿cómo? ¿se sale? ¿a dónde?

E5: que casi se acaban lo números

Sara: ¿y eso por qué?

E5 y E6 (juntos): Porque está calentísima

De acuerdo con lo anterior, los estudiantes han identificado el 10 como el número más pequeño, y las preguntas de Sara con respecto al 20 y al 40 ¿más grandes?, ¿mucho?, llevan a los estudiantes a la comparación mientras que las preguntas ¿cómo? ¿se sale? ¿a dónde? promueven la argumentación sobre el significado del número mayor en el contexto en que están trabajando. En esta línea, identificamos el conocimiento de Sara de la argumentación como parte de los procesos de razonamiento y demostración (C-PM).

Pasando a la etapa de presentación de los resultados, Sara dibuja un termómetro grande en un papel y lo coloca en la pizarra. Luego, un integrante de cada grupo se va acercando a registrar sus mediciones, cuidando no repetir los valores (Ilustración 3). Cuando todos los grupos han participado, Sara se dirige a la clase con preguntas que buscan que los estudiantes interpreten los resultados, como se observa en el siguiente fragmento:

Sara: Yo me pregunto... observando el termómetro [en la pizarra] ¿podríamos saber algo más sobre la temperatura del agua de la lluvia?

E5: Que es más que 11 y menos que 20, que está ahí.

Sara: ¡Oh! el intervalo entonces es de 11 a 20, es decir los números que están 
entre 11 y 20.

E5: sí, es menos fría que la de la nevera y menos caliente que el microondas.

Sara: Y eso ¿cómo es?

E5: Es templada, el agua de la lluvia es templada

Sara: ¿Sabéis algún número más templado?

E5: el 15.

Sara: ¿Por qué?

E5: Porque es más pequeño que el 20 y está ahí, por arriba del 11.

Figura 6 - Termómetro y resultado de las mediciones

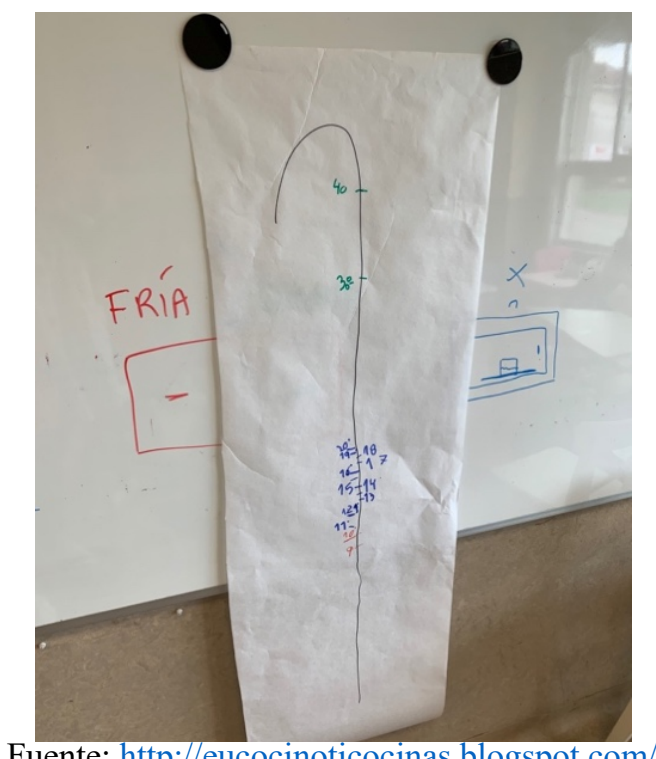

Fuente: http://eucocinoticocinas.blogspot.com/

De acuerdo con las respuestas de E5 y las intervenciones de otros estudiantes, la representación de las temperaturas en el termómetro (que hace las veces de una recta numérica vertical) permite a los estudiantes hacer ordenaciones y comparaciones. Esto también se observa cuando en la puesta en común el grupo describe tres intervalos, el 10 y valores menores que él corresponden al agua fría, los números entre el 11 y el 20 al agua templada y números entre 30 y 40 al agua caliente. Además, los estudiantes dejan de pensar que el agua de lluvia es más fría que el agua de la nevera ya que comprueban empíricamente que esta agua es templada. En línea con lo anterior, identificamos el conocimiento de Sara de que sus estudiantes aprenden a través de la experimentación, lo cual es parte de su conocimiento de las formas de aprendizaje de las matemáticas en la infancia (C-FAM).

Posteriormente, en el momento de comprobación y argumentación, Sara propone a 
los estudiantes sentir el agua en diferentes vasos y aproximarse con un número a la medida de su temperatura (Ilustración 4). Al inicio de la clase ya los estudiantes habían realizado estimaciones (de la temperatura del agua de lluvia) a partir de sus creencias y sensaciones, no obstante, en este momento de cierre, ellos cuentan con nueva información, la cual les permite reflexionar sobre sus respuestas tal como se observa en el siguiente fragmento:

Figura 7 - Estimaciones de la temperatura del agua

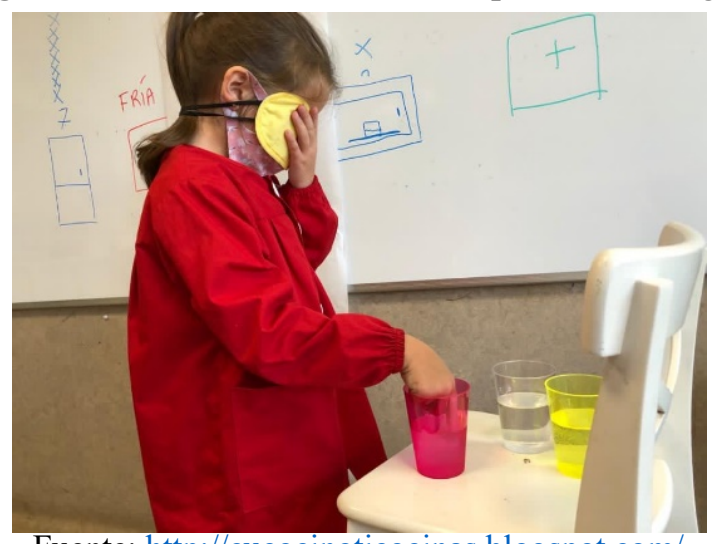

Fuente: http://eucocinoticocinas.blogspot.com/

Sara: toca y piensa de cuál de los tres vasos es esta agua.

E6: Del microondas.

Sara: ¿cómo es?, ¿cómo está?

E6: Caliente.

Sara: Y tu sabrías de que número se trata, si está caliente

E6: Uno grande, 40 era caliente y 40 es grande.

\section{Sara: Muy bien, ¿lo compruebas con el termómetro?}

E6: [mide y obtiene $30^{\circ}$ ] juy me he pasado!

A continuación, los estudiantes, reunidos en grupos, juegan a tratar de adivinar la temperatura de tres vasos de agua. Al proponer estas dos tareas una de estimación y comprobación y una de juego, Sara da cuenta de su conocimiento de la manipulación y el juego como una de las formas en que los niños aprenden matemáticas en la infancia (CFAM).

Sumando a lo anterior, durante la sesión de clases Sara considera los niveles de un Itinerario de Enseñanza de las Matemáticas. La maestra propone un contexto informal donde los estudiantes a partir de sus sensaciones clasifican y representan. Lo anterior, mediado por la manipulación de objetos cotidianos (vasos y agua) e instrumentos de 
medida (termómetro). Además, hay un contexto formal donde se presentan y discuten los resultados de las mediciones promoviendo la comparación y la argumentación. También se presenta un contexto intermedio donde nuevamente se parte de la experiencia sensorial, pero los datos obtenidos ahora se contrastan con lo aprendido en el momento de comprobación y argumentación. Al proponer estos tres contextos la profesora da cuenta de su conocimiento sobre la planificación y gestión de actividades de enseñanza de las matemáticas (C-PGA).

En la Tabla 1 se presenta un resumen de los subdominios identificados en el conocimiento de Sara:

Tabla 1 - Dominios y subdominios de conocimiento para enseñar matemáticas identificados en el episodio analizado.

\begin{tabular}{|c|c|c|}
\hline Dom & Subdominio & Descripción \\
\hline \multirow{3}{*}{$\begin{array}{l}\text { Conocimiento } \\
\text { Matemático en } \\
\text { Educación } \\
\text { Infantil (CM- } \\
\text { EI) }\end{array}$} & C-IeI & $\begin{array}{l}\text { Conocimiento de las cualidades sensoriales y el } \\
\text { papel que juegan en la comparación en } \\
\text { matemáticas. }\end{array}$ \\
\hline & $\mathrm{C}-\mathrm{CM}$ & $\begin{array}{l}\text { Conocimiento de la ordenación y comparación } \\
\text { (relación mayor y menor que) como contenidos } \\
\text { matemáticos en Educación Infantil. }\end{array}$ \\
\hline & C-PM & $\begin{array}{l}\text { Conocimiento de la argumentación como parte de } \\
\text { los procesos de razonamiento y demostración. } \\
\text { Conocimiento de la representación como proceso } \\
\text { matemático. }\end{array}$ \\
\hline \multirow{2}{*}{$\begin{array}{c}\text { Conocimiento } \\
\text { Didáctico de las } \\
\text { Matemáticas en } \\
\text { Educación } \\
\text { Infantil (CDM- } \\
\text { EI) }\end{array}$} & C-FAM & $\begin{array}{l}\text { Conocimiento de que la experimentación, la } \\
\text { manipulación y el juego son formas en que los } \\
\text { niños y niñas aprenden matemáticas en la infancia. }\end{array}$ \\
\hline & C-PGA & $\begin{array}{l}\text { Conocimiento de la planificación de las actividades } \\
\text { de enseñanza a partir de itinerarios. }\end{array}$ \\
\hline
\end{tabular}

\section{CONSIDERACIONES FINALES}

Desde hace algunas décadas, y como fruto del esfuerzo colectivo de diversos organismos y autores, las distintas agendas de investigación en educación matemática infantil han ido aportando datos acerca del análisis didáctico, el desarrollo profesional, la construcción y organización del conocimiento matemático o la práctica del profesor, entre otros aspectos (ALSINA, 2020b). En este estudio nos hemos focalizado en la práctica del profesor de infantil y su desarrollo profesional y, para seguir consolidando la disciplina, se 
ha presentado el modelo de Conocimientos para Enseñar Matemáticas en Educación Infantil (CEM-EI), que describe los conocimientos matemáticos y didácticos específicos que necesita el profesorado de esta etapa educativa para enseñar matemáticas (ALSINA y DELGADO, en revisión).

Desde este modelo de análisis, se han identificado los conocimientos para enseñar matemáticas que pone en juego una maestra de Educación Infantil durante un episodio de clase. De acuerdo con lo anterior, en el conocimiento de Sara se identificaron componentes matemáticos específicos del nivel de Educación Infantil correspondientes a todos los subdominios: C-IeI, C-CM y C-PM, lo cual es una evidencia de que la maestra posee un buen conocimiento disciplinar para enseñar matemáticas en esta etapa educativa, en sintonía con otro estudios preliminares que señalan también conocimientos sólidos del profesorado de infantil para enseñar esta materia (MUÑOZ-CATALÁN ET AL., 2019). De forma más concreta, cabe señalar que, en la realización del diagrama humano, por ejemplo, además del conocimiento de la representación como proceso matemático también se podría pensar en un conocimiento inicial de la maestra sobre lo que supone hacer una investigación estadística en Educación Infantil. Así, de forma todavía más específica, un posible tema a analizar sería el conocimiento de esta profesora para enseñar contenidos estadísticos en Educación Infantil.

En relación al conocimiento didáctico, se identificaron evidencias referentes a dos de los tres subdominos: C-FAM y C-PGA, lo cual sugiere que Sara conoce bien cómo aprenden matemáticas los niños y las niñas de infantil y qué planificación y gestión específica debe realizar en el aula para promover dicho aprendizaje. En esta línea, Hundeland et al. (2017) subrayan también la especificidad de la gestión en el aula de infantil para enseñar matemáticas. El único subdominio del conocimiento didáctico no identificado fue el conocimiento sobre las orientaciones curriculares (C-OCU), lo cual no significa que este componente no estuviera presente en el conocimiento de la maestra, sino que, con los datos recolectados, no se han podido hacer inferencias sobre el mismo. En este sentido, en futuras investigaciones la observación de clases podría complementarse con una entrevista a la maestra con el propósito de ampliar nuestra comprensión sobre su conocimiento e intentar encontrar evidencias de este subdominio.

En resumen, el análisis efectuado ha permitido identificar distintos subdominios tanto del CM-EI como del CDM-EI, lo cual podría ser un indicador de que el modelo CEM- 
EI es útil para analizar el conocimiento tanto matemático como didáctico del profesorado de Educación Infantil para enseñar matemáticas y, en consecuencia, los distintos dominios y subdominios describen adecuadamente dichos conocimientos, en la línea señalada por autores como Mosvold et al. (2011), que señalan la necesidad de modelos específicos para infantil. Sin embargo, estos primeros resultados a partir de la aplicación del modelo CEMEI deben tomarse con mucha cautela, por varios motivos: en primer lugar, porque se ha realizado un estudio de caso único, con lo cual los resultados obtenidos no se pueden generalizar; en segundo lugar, porque el perfil de la maestra que ha participado en el estudio no es el más representativo: si bien es cierto que hay una proporción importante del profesorado de infantil preocupado por mejorar sus prácticas, esta maestra posee el título de Doctora en Didáctica de las Matemáticas, lo que facilita el acceso a un amplio espectro de conocimientos provenientes de diversas teorías y enfoques de Educación Matemática; y, en tercer lugar, porque en este estudio se ha analizado un único episodio de clase, por lo que es obvio que, para empezar a extraer conclusiones, el modelo necesita más consistencia. Así, pues, aunque los primeros resultados obtenidos son alentadores, en el futuro será necesario diseñar nuevos estudios con otras maestras y maestros de Educación Infantil de diversos países que permitan ir afinando y consolidando el modelo CEM-EI para que, además ser un instrumento de análisis específico del conocimiento del profesorado infantil para enseñar matemáticas, sirva a este colectivo para identificar el conjunto de conocimientos necesarios para llevar a cabo esta labor y, con ello, promover su desarrollo profesional.

\section{REFERENCIAS}

ALSINA, Á. Más allá de los contenidos, los procesos matemáticos en Educación Infantil. Edma 0-6: Educación Matemática en la Infancia, v. 1 n. 1, p. 1-14, 2012.

ALSINA, Á. Matemáticas intuitivas e informales de 0 a 3 años. Elementos para empezar bien. Madrid: Narcea, S.A. de Ediciones, 2015.

ALSINA, Á. Seis lecciones de educación matemática en tiempos de cambio. Itinerarios didácticos para aprender más y mejor. Padres y Maestros, v. 376, p. 13-20, 2018.

ALSINA, Á. Estableciendo niveles de adquisición de conocimientos matemáticos importantes de 3 a 6 años: Rúbrica ACMI 3-6. Edma 0-6: Educación Matemática en la Infancia, v. 8 n. 2, p. 17-43, 2019a. 
Alsina, Á.; Delgado, R.

ALSINA, Á. Itinerarios didácticos para la enseñanza de las matemáticas (6-12 años). Barcelona: Editorial Graó, 2019b.

ALSINA, Á. La Matemática y su didáctica en la formación de maestros de Educación Infantil en España: crónica de una ausencia anunciada. La Gaceta de la Real Sociedad Matemática Española, v. 23 n. 2, p. 373-387, 2020a.

ALSINA, Á. Revisando la educación matemática infantil: una contribución al Libro Blanco de las Matemáticas. Edma 0-6, Educación Matemática en la Infancia, v. 9 n. 2, p. 1-20, $2020 b$.

ALSINA, Á. El Enfoque de los Itinerarios de Enseñanza de las Matemáticas: ¿por qué?, ¿para qué? y ¿cómo aplicarlo en el aula? TANGRAM - Revista de Educação Matemática, v. 3 n. 2, p. 127-159, 2020c.

ALSINA, Á. Cinco prácticas productivas para una enseñanza de las matemáticas a través de los procesos. Saber \& Educar, v. 28, p. 1-13, 2020d.

ALSINA, Á.; DELGADO, R. Modelo de Conocimientos para Enseñar Matemáticas en Educación Infantil. Acta Scientiae, Revista de Ensino de Ciências e Matemática, en revisión.

ALSINA, Á; MARTÍNEZ, M. La adquisición de conocimientos matemáticos intuitivos e informales en la Escuela Infantil: el papel de los materiales manipulativos. RELAdEI. Revista Latinoamericana de Educación Infantil, v.5, n.2, p. 127-136, 2016.

ALSINA, Á.; MAURANDI, A.; FERRE, E.; CORONATA, C. Validating an Instrument to Evaluate the Teaching of Mathematics Through Processes. International Journal of Science and Mathematics Education, v. 19, p. 559-577, 2021.

BALL, D.; THAMES, M. H.; PHELPS, G. Content knowledge for teaching: What makes it special? Journal of Teacher Education, v. 59 n. 5, p. 389-407, 2008.

BROWN, R.D. Neuroscience of Mathematical Cognitive Development: From Infancy Through Emerging Adulthood. Cham: Springer, 2018.

BAROODY, A.J. Children's Mathematical Thinking. A developmental framework for preschool, primary, and special education teachers. New York, US: Teachers College Press, 1987.

CARRILLO, J.; CLIMENT, N.; MONTES, M.; CONTRERAS, L.; FLORES-MEDRANO, E.; ESCUDERO-ÁVILA, D.;... MUÑOZ-CATALÁN, M ${ }^{\mathrm{a}}$.C. ET AL. The mathematics teacher's specialised knowledge (MTSK) model. Research in Mathematics Education, v. 20, p. 236-253, 2018.

CHARALAMBOUS, C.; PITTA-PANTAZI, D. Perspectives on priority mathematics education: Unpacking and understanding a complex relationship linking teacher knowledge, teaching, and learning. En: L. ENGLISH; D. KIRSHNER (Eds.), Handbook of international research in mathematics education. New York: Routledge, p. 19-59, 2016.

CLEMENTS, H. D.; SARAMA, J. Learning and teaching early math: The learning trajectories approach. New York: Routledge, 2009. 
CROSS, C. T.; WOODS, T. A.; SCHWEINGRUBER, H. (Eds.). Mathematics learning in early childhood: Paths toward excellence and equity. Washington, DC, US: National Academies Press, 2009.

GEIST, E. Children are born mathematicians: supporting mathematical development, birth to age 8. Merrill/Pearson, 2014.

GODINO, J.; GIACOMONE, B.; BATANERO, C.; FONT, V. Enfoque ontosemiótico de los conocimientos y competencias del profesor de matemáticas. Bolema, v. 31 n. 57, p. 90$113,2017$.

HARGREAVES, A.; EARL, L.; MOORE, S.; MANNING, S. Aprender a cambiar. La enseñanza más allá de las materias y los niveles. Barcelona: Editorial Octaedro, 2001.

HUNDELAND, P.S., ERFJORD, I., Y CARLSEN, M. A kindergarten teacher's revealed knowledge in orchestration of mathematical activities. En: T. Dooley, y G. Gueudet (Eds.). Proceedings of the CERME 10. DCU Institute of Education and ERME, pp. 1853 - 1860, 2017.

KRIPPENDORFF, K. Content analysis: An introduction to its methodology. Sage publications, 2004

MALAGUZZI, M. La educación infantil en Reggio Emilia. Rosa Sensat-Octaedro, 2001

MONTESSORI, M. La mente absorbente del niño. México, D.F., México: Editorial Diana, 1986.

MOSVOLD, R., BJULAND, R., FAUSKANGER, J., y JAKOBSEN A. Similar but different -investigating the use of MKT in a Norwegian kindergarten setting. En: M. Pytlak, T. Rowland, y E. Swoboda (Eds.). Proceedings of the CERME 7, University of Rzeszów, pp. 1802-1811, 2011.

MUÑOZ-CATALÁN, C., JOGLAR, N., RAMÍREZ, M., ESCUDERO, A.M., AGUILAR, A. Y RIBEIRO, M. El conocimiento especializado del profesor de infantil desde el aula de matemáticas. En E. Badillo, N. Climent, C. Fernández y M. T. González (Eds.), Investigación sobre el profesor de matemáticas: formación, práctica de aula, conocimiento y competencia profesional. Ediciones Universidad Salamanca, pp. 63-84, 2019.

PIAGET, J. L'équilibration des structures cognitives. Problème central du développement. Presses Universitaries de France, 1975

PIKLER, E. Moverse en libertad. Desarrollo de la motricidad global. Madrid, España: Narcea, 1985.

PONTE, J.P. Estudos de caso em educação matemática. Bolema, v. 25, p.105-132, 2006.

NATIONAL ASSOCIATION FOR THE EDUCATION OF YOUNG CHILDREN AND NATIONAL COUNCIL FOR TEACHERS OF MATHEMATICS [NAEYC y NCTM] Early childhood mathematics: Promoting good beginnings. A joint position statement. Recuperado de http://www.naeyc.org/files/naeyc/file/positions/psmath.pdf, 2002.

NATIONAL COUNCIL OF TEACHERS OF MATHEMATICS [NCTM]. Principles y Standards for School Mathematics. Reston, VA, US: National Council of Teachers of Mathematics, 2000. 
NOLLA, Á.; MUÑOZ, R.; CERISOLA, A.; FERNÁNDEZ, B. La formación inicial de los maestros en matemáticas y su didáctica. Revista Interuniversitaria de Formación del Profesorado, v. 96 n. 35.1, p. 185-208, 2021.

ROWLAND, T.; HUCKSTEP, P.; THWAITES, A. Elementary teachers' mathematics subject knowledge: The knowledge quartet and the case of Naomi. Journal of Mathematics Teacher Education, v. 8 n. 3, p. 255-281, 2005.

SÁMUEL, M., VANEGAS, Y., y GIMÉNEZ, J. Caracterización del conocimiento matemático de futuras maestras de Educación Infantil. Bordón. Revista de Pedagogía, v. 70, n.3, 61-75, 2018.

SHULMAN, L.S. Those who understand: Knowledge growth in teaching. Educational researcher, v. 15 n. 2, p. 4-14, 1986. 